\title{
Electron loss from medium-energy projectiles in collisions with heavy target atoms $\dagger$
}

\author{
D H Jakubassa \\ Institute of Physics, University of Oslo, Oslo, Norway
}

Received 2 November 1979

\begin{abstract}
The energy and angular distribution of projectile electrons emitted in asymmetric collisions is calculated within the quantum mechanical electron impact approximation. The rise of the differential cross section in the backward direction is shown to follow closely the behaviour of electrons elastically scattered from the target. As an example, $(\mathrm{H}, \mathrm{Ne})$ and $(\mathrm{H}, \mathrm{Ar})$ collisions are studied and the results are compared with experiment.
\end{abstract}

\section{Introduction}

The ionisation of projectiles in heavy-ion collisions has been extensively studied in connection with the determination of the average charge state of a projectile traversing a target (Betz 1972). Apart from total cross section measurements also differential cross sections have been investigated in order to obtain more information about the colliding system. The momentum distribution of the initial electronic state, for example, can be deduced from an observation of the energy distribution of the emitted electrons (Burch et al 1973). Experiments have now also been carried out on the angular distribution of the electrons (Duncan and Menendez 1979), which can be used as a tool to study the properties of the target atom.

For the calculation of the electron loss the first-order Born approximation can be applied provided that the velocity $v$ of the projectile is larger than the orbiting velocity $v_{\mathrm{e}}$ of any of the electrons of the projectile-target system. Thereby one usually includes the contribution from the excitation of target electrons (Bates and Griffin 1955) by means of a closure approximation. For these high projectile velocities, the angular distribution of the emitted electrons shows a smooth decrease with angle (Drepper and Briggs 1976). If, however, $v<v_{\mathrm{e}}$ a rise of the differential cross section in the backward direction is observed (Duncan and Menendez 1979) which cannot be reproduced by the Born approximation even if one includes the second-order term. To explain this behaviour the binary encounter approximation (elastic scattering model) has been used where the projectile electron is described as a free electron which scatters elastically from the target, and the corresponding cross section is then weighted with the velocity distribution of the electron in its initial state (Burch et al 1973). Actually, in this approach there are also deviations from the data both in the angular and energy distribution of the electrons.

† This work was supported by the Deutsche Forschungsgemeinschaft. 
An advantage of the quantum mechanical description over the binary encounter approach is the correct incorporation of momentum conservation in the whole system. There are two possibilities for an approximate treatment of the three-body problem, depending on the nuclear charges. In systems where the projectile (charge $Z_{1}$ ) is heavier than the target $\left(Z_{2}\right)$ the electron loss can be described by ionisation in the rest system of the projectile followed by a transformation to the laboratory (target) system. The final electronic state is thereby an eigenstate to the projectile (Drepper and Briggs 1976). On the other hand, if the target field dominates the projectile field $\left(Z_{1}<Z_{2}\right)$ the electron loss is more readily expressed as charge transfer to the continuum. Thereby it is important to go beyond the first-order Born approximation. An adequate way to include higher-order terms in the target interaction is given by the electron impact approximation which is the quantum mechanical analogy of the binary encounter approach. An additional inclusion of one interaction with the projectile leads to the impulse approximation (McDowell and Coleman 1970) which determines the highvelocity behaviour for charge transfer (Briggs 1977).

In this paper the cross section $\mathrm{d}^{2} \sigma / \mathrm{d} E_{f} \mathrm{~d} \Omega_{f}$ for electron loss differential in electron energy and angle is calculated within the electron impact approximation which is easier to handle than the impulse approximation. It should hold for strongly asymmetric systems with intermediate projectile velocities such that $\frac{1}{2} m v^{2}(m=$ electron mass) is larger than the electronic binding energy in the initial state, and comparable with the excitation energies in the target. Section 2 contains the derivation of the cross section, where the elastic electron-scattering cross section is used to describe the interaction between projectile electron and target. Effects of inelastic electron scattering are taken into account in $\S 3 . \mathrm{d}^{2} \sigma / \mathrm{d} E_{f} \mathrm{~d} \Omega_{f}$ is evaluated in the cases of $\mathrm{H}$ impact on Ne and $\mathrm{Ar}$ $(\S 4)$ followed by a comparison with experiment and discussion $(\$ 5)$.

\section{Electron loss in the electron impact approximation}

We proceed along the lines previously developed in connection with radiative ionisation (Jakubassa and Kleber 1975). As we consider systems with $Z_{1} \ll Z_{2}$ the ejected electron is described by an eigenstate to the target. The electron impact approximation can be obtained by starting from the transition matrix element in the plane-wave Born approximation

$$
a_{f i}=\left\langle\chi_{f}\left(\boldsymbol{R}_{f}\right) \psi_{f}(\boldsymbol{r})\left|V_{\mathrm{T}}(\boldsymbol{r})\right| \chi_{i}\left(\boldsymbol{R}_{i}\right) \psi_{i}\left(\boldsymbol{r}_{i}\right)\right\rangle
$$

where $\chi_{i, f}$ are the nuclear and $\psi_{i, f}$ the electronic wavefunctions and $\boldsymbol{R}_{i, f}$ the centre of mass coordinates in initial and final state (figure 1). $V_{\mathrm{T}}(\boldsymbol{r})$ stands for the interaction between projectile electron and target.

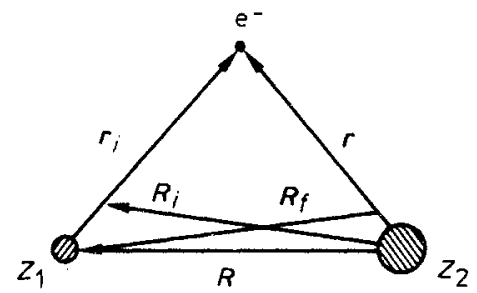

Figure 1. Coordinate system for a three-body problem consisting of projectile $\left(Z_{1}\right)$, target $\left(Z_{2}\right)$ and electron. $\boldsymbol{R}_{i, f}$ connects one particle with the centre of mass of the other two particles. 
Introducing the Fourier transform $\phi_{i}\left(\boldsymbol{k}_{i}\right)$ of the initial electronic state $\psi_{i}\left(\boldsymbol{r}_{i}\right)$ one can perform the integration over $\boldsymbol{R}$ and express (2.1) by electronic properties alone

$$
\begin{aligned}
a_{f i} & =\phi_{i}\left(\boldsymbol{k}_{i}\right)(2 \pi)^{-3 / 2} \int \mathrm{d} \boldsymbol{r} \psi_{f}^{*}(\boldsymbol{r}) V_{\mathrm{T}}(\boldsymbol{r}) \mathrm{e}^{\mathrm{i}\left(\boldsymbol{k}_{i}+m v / \hbar\right) \boldsymbol{r}} \\
& =-\frac{\hbar^{2}}{m}(2 \pi)^{-2} \phi_{i}\left(\boldsymbol{k}_{i}\right) f\left(\boldsymbol{k}_{i}+m \boldsymbol{v} / \hbar, \boldsymbol{k}_{f}, \theta_{i f}\right)
\end{aligned}
$$

where we introduced the scattering amplitude $f$ for the scattering of a plane wave with momentum $\hbar \boldsymbol{k}_{i}+m \boldsymbol{v}$ into a final state with momentum $\hbar \boldsymbol{k}_{f}$. $\theta_{i f}$ is the scattering angle (between $\boldsymbol{k}_{i}+m \boldsymbol{v} / \hbar$ and $\boldsymbol{k}_{f}$ ). When $\psi_{f}(\boldsymbol{r})$ is described by a plane wave, $f$ represents the first-order Born approximation for an electron scattered from the target potential $V_{\mathrm{T}}(\boldsymbol{r})$. For strong potentials, however, one has to include higher orders in the perturbation. This is easily done by replacing the incident plane wave by the exact scattering state $\hat{\psi}_{i}(\boldsymbol{r})$ of the target with initial momentum $\hbar \boldsymbol{k}_{i}+m v$ such that $f$ becomes

$$
f=-(2 \pi)^{2} \frac{m}{\hbar^{2}} \int \mathrm{d} \boldsymbol{r} \psi_{f}^{*}(\boldsymbol{r}) V_{\mathrm{T}}(\boldsymbol{r}) \hat{\psi}_{i}(\boldsymbol{r}) .
$$

Inserting (2.3) into (2.2) one arrives at the electron impact approximation: the projectile electron behaves as quasi-free in the target field and the initial momentum is weighted by its probability amplitude $\phi_{i}\left(\boldsymbol{k}_{i}\right)$. The difference to the elastic scattering model lies in the fact that besides momentum conservation in the projectile-target system, the electron impact approximation allows for inelastic scattering via (2.3). The differential cross section for electron loss is given by

$$
\mathrm{d} \sigma=\frac{(2 \pi)^{4}}{\hbar v}\left|a_{f i}\right|^{2} \delta\left(\epsilon_{f}-\epsilon_{i}\right) \mathrm{d} \boldsymbol{k}_{f} \mathrm{~d} \boldsymbol{K}_{f}
$$

where $\boldsymbol{K}_{f}$ is the wavenumber of the relative nuclear motion in the final state. Using momentum conservation we can eliminate the nuclear quantities and write

$\frac{\mathrm{d}^{2} \sigma}{\mathrm{d} E_{f} \mathrm{~d} \Omega_{f}}=\frac{\hbar \boldsymbol{k}_{f}}{m v} \int \mathrm{d} \boldsymbol{k}_{i}\left|\phi_{i}\left(\boldsymbol{k}_{i}\right)\right|^{2} \frac{\mathrm{d} \sigma_{\mathrm{e}}}{\mathrm{d} \Omega}\left(\boldsymbol{k}_{i}+m \boldsymbol{v} / \hbar, \boldsymbol{k}_{f}, \theta_{i f}\right) \boldsymbol{\delta}\left(E_{f}-E_{i}-\frac{1}{2} m v^{2}-\hbar \boldsymbol{v} \boldsymbol{k}_{i}\right)$

where we introduced the electron scattering cross section $\mathrm{d} \sigma_{\mathrm{e}} / \mathrm{d} \Omega=|f|^{2} . E_{i}$ is the energy of the initial electronic state and $E_{f}=\hbar^{2} k_{f}^{2} / 2 m$. In order to establish the connection between the electron loss cross section and the electron scattering cross section we make use of the fact that the momentum distribution $\left|\phi_{i}\left(\boldsymbol{k}_{i}\right)\right|^{2}$ is strongly peaked for $k_{i} \rightarrow 0$ such that we can take $\mathrm{d} \sigma_{\mathrm{e}} / \mathrm{d} \Omega$ outside the integral at the minimum value $k_{i}=k_{i z} e_{z}$ where $k_{i z}$ is given by energy conservation and $v$ is chosen as $z$ direction. The remaining integral yields the Compton profile $J_{i}\left(k_{i z}\right)$ (which equals $(8 / 3 \pi) a_{i}\left(k_{i z}^{2} a_{i}^{2}+1\right)^{-3}$ with $a_{i}=\hbar^{2} /\left(m Z_{1} e^{2}\right)$ for the hydrogenic 1 s state $)$. In this peaking approximation $\theta_{i f}$ reduces to the angle $\theta_{f}$ (between $k_{f}$ and $v$ ) i.e. the ejection angle of the projectile electron. We obtain

$$
\begin{aligned}
& \frac{\mathrm{d}^{2} \sigma}{\mathrm{d} E_{f} \mathrm{~d} \Omega_{f}}=\frac{k_{f}}{m v^{2}} J_{i}\left(k_{i z}\right) \frac{\mathrm{d} \sigma_{e}}{\mathrm{~d} \Omega}\left(\left(k_{i z}+m v / \hbar\right) \boldsymbol{e}_{z}, \boldsymbol{k}_{f}, \theta_{f}\right) \\
& k_{i z}=\frac{1}{\hbar v}\left(E_{f}-E_{i}-\frac{1}{2} m v^{2}\right) .
\end{aligned}
$$

We now proceed with the calculation of the electron scattering cross section. The replacement of the state $\hat{\psi}_{i}(\boldsymbol{r})$ by a Coulomb wave only gives good results if the average 
electron energy $\frac{1}{2} m v^{2}$ is larger than the 1s binding energy of the target (Jhanwar et al 1978) since it leads to a smooth decrease of $f$ with angle. If, however, $\frac{1}{2} m v^{2}$ coincides with excitation energies in the target then the resulting resonances and interference effects can only be described by an exact solution of the Schrödinger equation.

An exact evaluation of the scattering amplitude (2.3) is very involved. Actually, if $\frac{1}{2} m v^{2}$ is much larger than the initial binding energy $E_{i}$ the difference $\left|\boldsymbol{k}_{i}+m \boldsymbol{v} / \hbar\right|-k_{f}$ is small compared with $k_{f}$ in the $E_{f}$ region where the cross section (2.6) is large. Then, as a first approximation, one may obtain $f$ by means of the elastic scattering theory. Inelastic effects will be discussed later.

In order to find the elastic scattering amplitude we use the method of partial waves. In this approach, $f$ can be expressed in terms of the phaseshifts $\delta_{l}$ which are obtained from the asymptotic behaviour of the wavefunctions obeying the radial part of the Schrödinger equation

$$
\frac{\hbar^{2}}{2 m}\left(\frac{\mathrm{d}^{2}}{\mathrm{~d} r^{2}}+k^{2}-\frac{l(l+1)}{r^{2}}-\frac{2 m}{\hbar^{2}} V_{\mathrm{T}}(r)\right) R_{l}(r)=0
$$

with $k=k_{i z}+m v / \hbar$. The scattering potential $V_{\mathrm{T}}(r)$ is chosen to consist of a term due to static screening, $V_{\mathrm{sc}}$, and one due to polarisation, $V_{\mathrm{pol}}$ (exchange effects are small in the velocity region considered here and therefore neglected)

$$
V_{\mathrm{T}}(r)=-\frac{Z_{2} e^{2}}{r} \sum_{i=1}^{2}\left(a_{i} \mathrm{e}^{-\alpha_{i} r}+b_{i} r \mathrm{e}^{-\beta_{i} r}\right)-\frac{\alpha r^{2}}{2\left(r^{2}+d^{2}\right)^{3}} .
$$

The constants $a_{i}, b_{i}, \alpha_{i}$ and $\beta_{i}$ are fitted to a Hartree-Fock potential (Strand and Bonham 1964), $\alpha$ is the dipole polarisability (Fraga et al 1976) and the screening constant $d$ equals $3 \hbar^{2} \mathrm{k} /(8 \mathrm{~m} \Delta)$ where $\Delta$ is the mean excitation energy of the target. We take $\Delta=2.687 e^{4} m / \hbar^{2}$ for Ne and $0.93 e^{4} m / \hbar^{2}$ for $\operatorname{Ar}$ (which roughly corresponds to the $\mathrm{s}$ binding energy of the outermost shell).

When the scattering energy is comparable to inner-shell binding energies many partial waves are needed to obtain convergence. Following Jhanwar et al (1978) one can reduce the numerical efforts by recalling that for high angular momenta $l>N$ $(N \sim 10$ for $\mathrm{Ne}$ and $\sim 15$ for $\mathrm{Ar})$ the phaseshifts are only determined by the polarisation potential and can be calculated (analytically) in the Born approximation. Thus it is only necessary to solve (2.7) for $l \leqslant N$. The contribution to $f$ for $l>N$ is then obtained by calculating the scattering amplitude $f_{\mathrm{pol}}$ due to $V_{\mathrm{pol}}$ in Born approximation $\left(f_{\mathrm{pol}}=\right.$ $\left.\pi \alpha m \mathrm{e}^{-x}(3-x) /\left(16 \hbar^{2} d\right), x=2 k d \sin \frac{1}{2} \theta\right)$ and subtracting the corresponding $N$ lowest $l$-expansion terms given by the phaseshifts $\eta_{l}^{\text {pol }}$ :

$$
f=\frac{1}{k} \sum_{l=0}^{N}(2 l+1) P_{l}(\cos \theta)\left(\mathrm{e}^{\mathrm{i} \delta_{l}} \sin \delta_{l}-\eta l^{\mathrm{pol}}\right)+f_{\mathrm{pol}} .
$$

When calculating $\delta_{l}$ from the large $r$ behaviour of $R_{l}(r)$ the convergence is appreciably speeded up if one first determines the phaseshifts belonging to $V_{\mathrm{T}}=0$ at fixed $r$ (they vanish for $r \rightarrow \infty$ ) and subtracts them from the phaseshifts of $V_{\mathrm{T}}(r)$ to obtain $\delta_{l}$ at $r$. We compared $|f|^{2}$ with the experimental electron scattering cross section (Jhanwar et al 1978, DuBois and Rudd 1975) in the region $k \sim m v / \hbar$ and found good agreement.

\section{Inelastic effects}

The calculation of the inelastic electron scattering within the electron impact approximation is very complicated since an exact evaluation of the scattering amplitude (2.3) 
involves multi-electron processes. A possibility of describing the inelastic scattering in the one-electron model is given by the impulse approximation, which can be obtained from the post form of the plane-wave Born approximation $\left((2.1)\right.$ with $V_{\mathrm{T}}(\boldsymbol{r})$ replaced by $V_{\mathrm{P}}\left(\boldsymbol{r}_{i}\right)$ ) along the lines used in $\S 2$. In this approximation, the double-differential electron loss cross section turns out to be

$$
\begin{aligned}
& \frac{\mathrm{d}^{2} \sigma}{\mathrm{d} E_{f} \mathrm{~d} \Omega_{f}}=\frac{2 \pi m \boldsymbol{k}_{f}}{\hbar^{3} v} \int \mathrm{d} \boldsymbol{k}_{0} \delta\left(E_{f}-E_{i}-\frac{1}{2} m v^{2}-\hbar v \boldsymbol{k}_{0}\right) \\
& \quad \times\left|\int \mathrm{d} \boldsymbol{k}_{i} \phi_{i}\left(\boldsymbol{k}_{i}\right) V_{\mathrm{P}}\left(\boldsymbol{k}_{0}-\boldsymbol{k}_{i}\right) \int \mathrm{d} \boldsymbol{r} \psi_{f}^{*}(\boldsymbol{r}) \exp \left[\mathrm{i}\left(\boldsymbol{k}_{0}-\boldsymbol{k}_{i}\right) \boldsymbol{r}\right] \hat{\psi}_{i}\left(\boldsymbol{k}_{i}+m \boldsymbol{v} / \hbar, \boldsymbol{r}\right)\right|^{2}
\end{aligned}
$$

where $V_{\mathrm{P}}\left(\boldsymbol{k}_{0}-\boldsymbol{k}_{i}\right)$ is the Fourier transform of the projectile field and $\psi_{f}$ and $\hat{\psi}_{i}$ are both exact scattering functions of the target with momentum $\hbar \boldsymbol{k}_{f}$ and $\hbar \boldsymbol{k}_{i}+m \boldsymbol{v}$, respectively. The additional coupling to the projectile field introduces thereby an extra integral over momentum which complicates the evaluation considerably. We therefore restrict ourselves to give an estimate of the inelastic effects within the electron impact approximation.

When inserting the elastic scattering cross section into (2.6) we find that in this approximation the angular distribution of the emitted projectile electrons is just given by the electron scattering cross section. In order to estimate the corrections originating from $k_{f}$ being unequal to $k_{i z}+m v / \hbar$ we recall that the inelastic scattering cross section depends only on the momentum transfer $q_{\text {in }}=\left|\boldsymbol{k}_{f}-\left(k_{i z} \boldsymbol{e}_{z}+m \boldsymbol{v} / \hbar\right)\right|$ and the angle between $q_{\text {in }}$ and $k_{i z} e_{z}+m v / \hbar$, where the dependence on $q_{\text {in }}$ is the dominating one, while the elastic cross section depends only on $q_{\mathrm{el}}=2 k \sin \frac{1}{2} \theta$. So we incorporate the main part of the inelastic effects by making the substitution $q_{\mathrm{el}} \rightarrow q_{\mathrm{in}}$

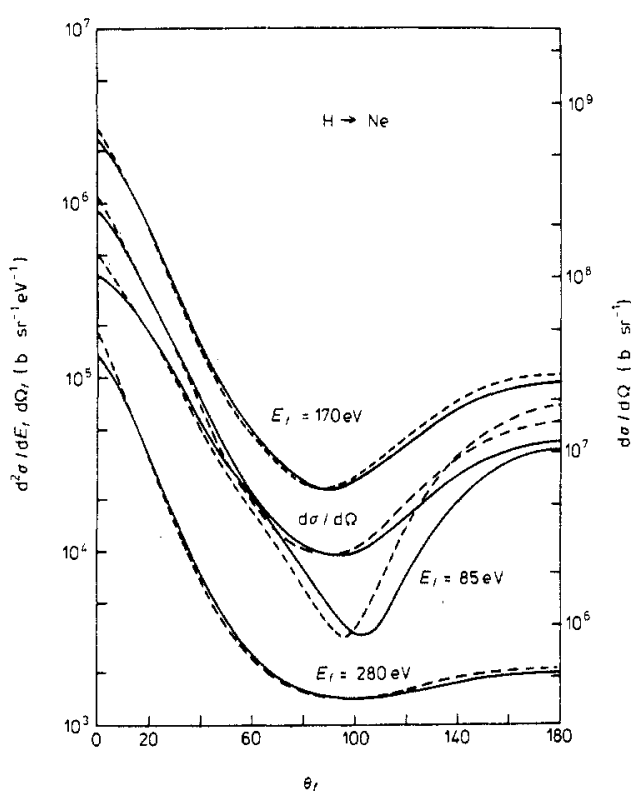

Figure 2. Double-differential cross section for electron loss in $(\mathrm{H}, \mathrm{Ne})$ collisions with impact energy $0.31 \mathrm{MeV}$ as a function of emission angle $\theta_{f}$ with the emission energy $E_{f}$ as a parameter. Also shown is the single-differential cross section (right-hand scale). Broken curves are calculations using the elastic electron-target scattering cross section, full curves include inelastic effects (via equation (3.2)). 
i.e.

$$
\sin \frac{1}{2} \theta \rightarrow \frac{1}{2 k}\left(k_{f}^{2}+k^{2}-2 k_{f} k \cos \theta_{f}\right)^{1 / 2} \quad k=k_{i z}+m v / \hbar
$$

in the scattering amplitude (2.9). This reduces the electron loss cross section mainly near $0^{\circ}$ and in the backward direction, and shifts the minimum to slightly larger angles as can be seen from figures 2 and 3 where the double-differential electron loss cross section is displayed as a function of electron emission angle $\theta_{f}$.

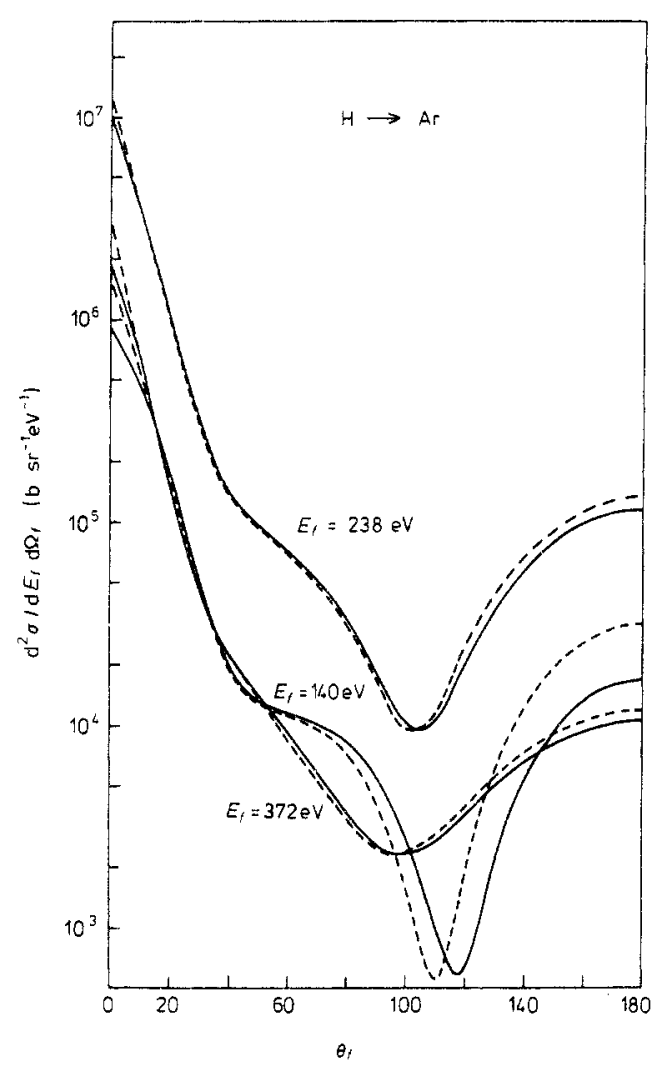

Figure 3. Double-differential cross section for electron loss in $(\mathrm{H}, \mathrm{Ar})$ collisions with impact energy $0.5 \mathrm{MeV}$ as a function of emission angle $\theta_{f}$ for various emission energies $E_{f}$. Broken and full curves have the same meaning as in figure 2 .

\section{Electron loss in the case of $H \rightarrow N e$ and $H \rightarrow A r$ collisions}

We consider collisions with a projectile energy of $0.31 \mathrm{MeV}$ for $\mathrm{Ne}$ targets (corresponding to $\frac{1}{2} m v^{2}=170 \mathrm{eV}=-1 / 8 E_{1 \mathrm{~s}}(\mathrm{Ne})$ ) and of $0.5 \mathrm{MeV}$ in the argon case (i.e. $\left.\frac{1}{2} m v^{2}=272 \cdot 4 \mathrm{eV}=-1 / 16 E_{1 \mathrm{~s}}(\mathrm{Ar})\right)$. For these conditions the electron impact approximation should be well suited.

From (2.6) it follows that the projectile electrons are mainly ejected with velocity around $v$ since then $k_{i z}$ attains its minimum value. This well known fact is shown in figure 4. Actually the peak energy $E_{\max }$ is not only shifted slightly due to the binding energy $\left|E_{i}\right|$ but is also dependent on the emission direction $\theta_{f}$. While for high projectile energies (where the Born approximation is valid) one finds a monotonous decrease with $\theta_{f}$ (Drepper and Briggs 1976) the dependence on $\theta_{f}$ shows structure for medium-energy 

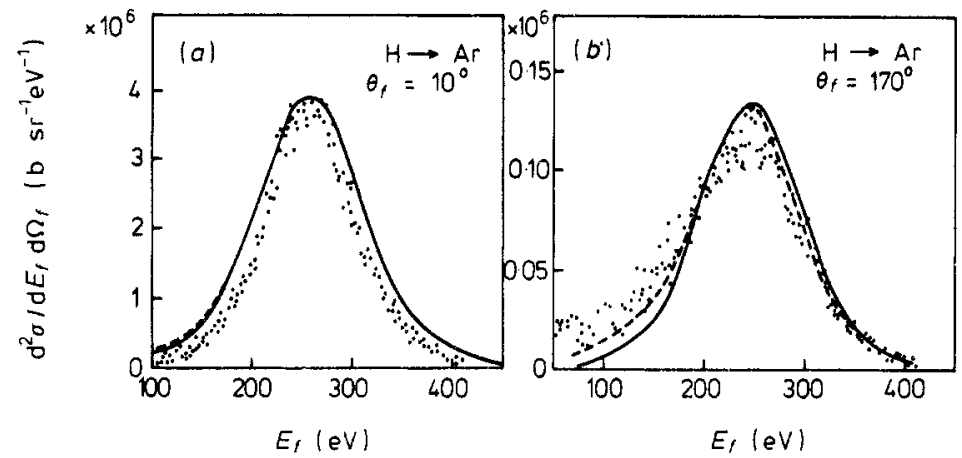

Figure 4. Double-differential cross section for electron loss in ( $\mathrm{H}, \mathrm{Ar})$ collisions with impact energy $0.5 \mathrm{MeV}$ as function of the emission energy $E_{f}$ at $\theta_{f}=10^{\circ}(a)$ and $170^{\circ}(b)$. Broken and full curves have the same meaning as in figure 2 . The 'inelastic' curve at $170^{\circ}$ is multiplied by a factor 1.2 to give the same peak height. The data are from Duncan $e t$ al (1979) and are normalised to theory.

projectiles (figure 5) which is the larger the lower $\frac{1}{2} m v^{2}$ is compared with $\left|E_{1 \mathrm{~s}}\right|$. This originates from the rapid change of the electron scattering cross section with energy and angle and is thus a target effect.

The cross section integrated over energy, $\mathrm{d} \sigma / \mathrm{d} \Omega_{f}$, is shown in figure 2 . Its angular dependence follows closely the double-differential cross section near the peak energy. In order to study the velocity dependence of $\mathrm{d} \sigma / \mathrm{d} \Omega_{f}$ we therefore plotted $\mathrm{d}^{2} \sigma / \mathrm{d} E_{f} \mathrm{~d} \Omega_{f}$ at $E_{f}=\frac{1}{2} m v^{2}$ in figure 6 for various velocities $v$. For increasing $v$ the backward rise of the cross section becomes weaker which is correlated to the behaviour of the elastic electron scattering cross section. For comparison we show also the Born result (obtained with the potential (2.8)) for the highest velocity, which overestimates the cross section for small angles and lies below the present result for large $\theta_{f}$. In all our calculations we used the peaking approximation (2.6). The deviations from the result
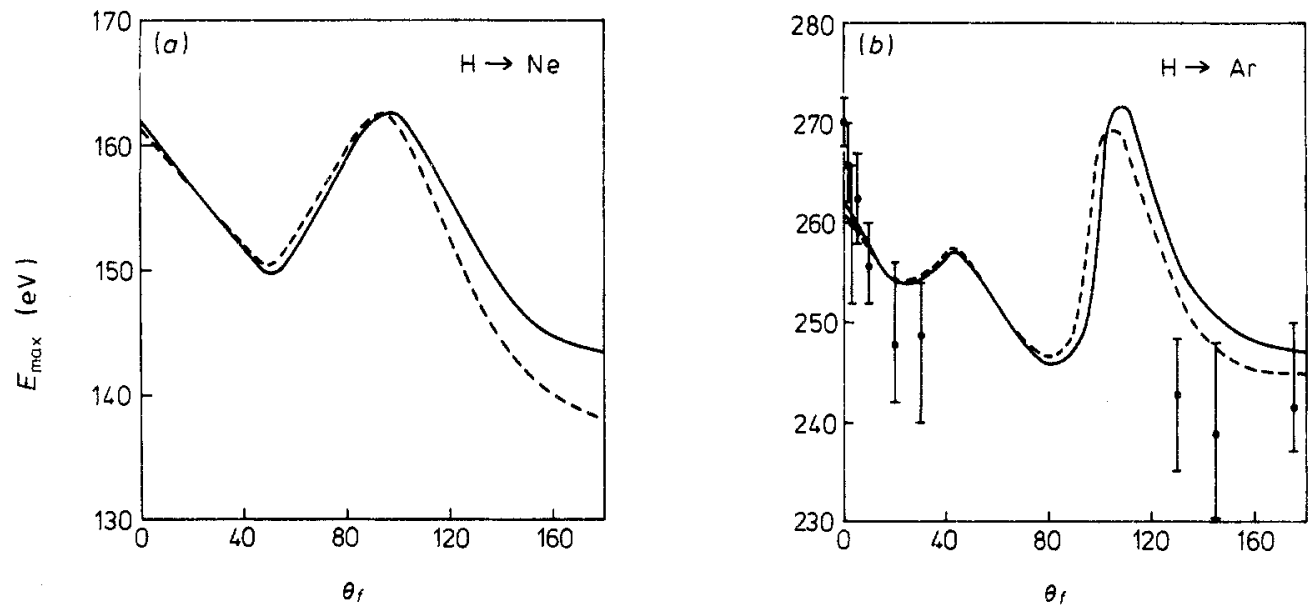

Figure 5. Peak energy $E_{\max }$ of the emission spectrum for $(\mathrm{H}, \mathrm{Ne})$ collisions $(a)$ at $0.31 \mathrm{MeV}$ and for $(\mathrm{H}, \mathrm{Ar})$ collisions $(b)$ at $0.5 \mathrm{MeV}$ as a function of emission angle $\theta_{f}$. Broken and full curves have the same meaning as in figure 2 . The data are from Duncan et al (1979). 


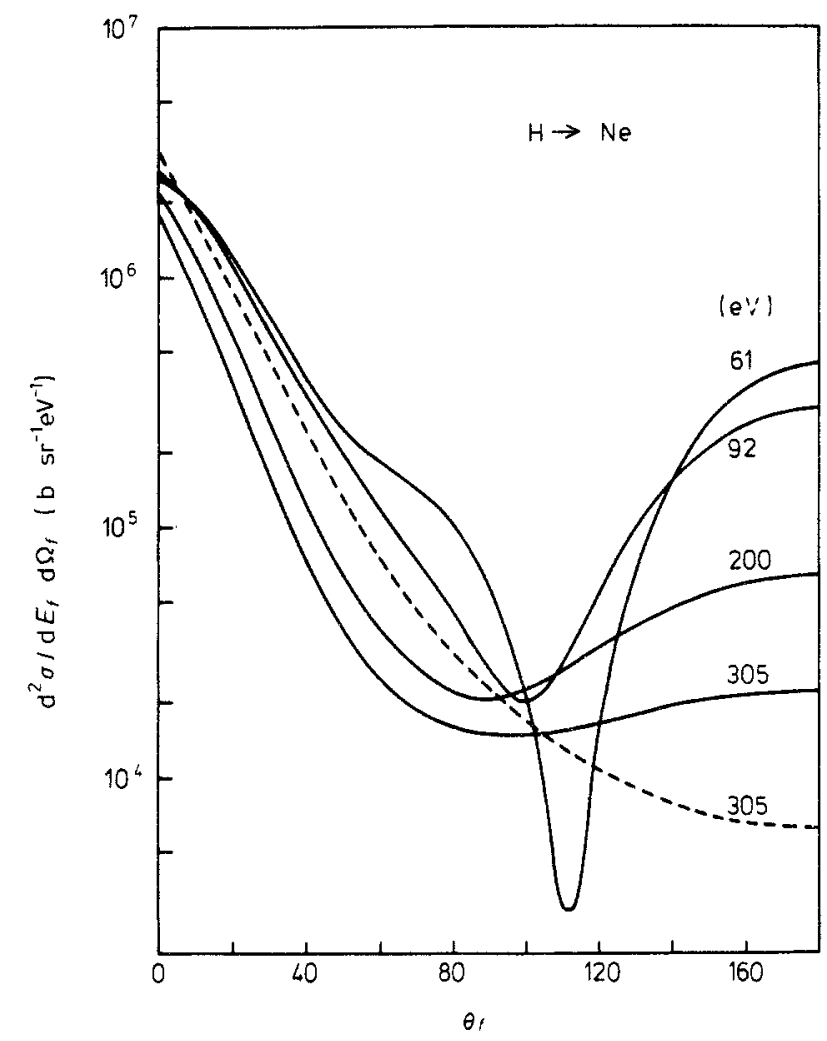

Figure 6. Double-differential cross section for electron loss in $(\mathrm{H}, \mathrm{Ne})$ collisions as a function of emission angle $\theta_{f}$ where the emission energy $E_{f}$ equals $\frac{1}{2} m v^{2}$. The velocity dependence is indicated by choosing $\frac{1}{2} m v^{2}$ as a parameter. The full curves are calculations including inelastic effects, the broken curve shows the Born approximation for comparison.

obtained with (2.5) are very small except for $E_{f} \ll \frac{1}{2} m v^{2}$ (where also the inelastic effects become more important) and for small angles $\theta_{f} \leqslant 10^{\circ}$. At $\theta_{f}=0$ the peaking approximation overestimates the cross section by a factor of two.

\section{Comparison with experiment and discussion}

Recently detailed experiments have been performed on projectile electron emission in collisions of $\mathrm{H}$ with $\mathrm{Ar}$ at $0.5 \mathrm{MeV}$. Duncan and Menendez (1979) measured the angular distribution of the emitted electrons and found a strong rise in the backward direction which they tried to explain with the elastic scattering model. Figure 7 shows their results together with our calculation. Although our theory reproduces the qualitative features there exist deviations. There is no great difference between the electron impact approximation and the elastic scattering model in the angular distribution since it is in both cases determined by the electron-target interaction. Actually part of the deviations between the two theories may result from a different evaluation of the elastic electron scattering cross section. The discrepancies between theory and experiment cannot be explained with inaccuracies in calculating the electron scattering cross section or with a slowing down of the projectile electron before scattering. 


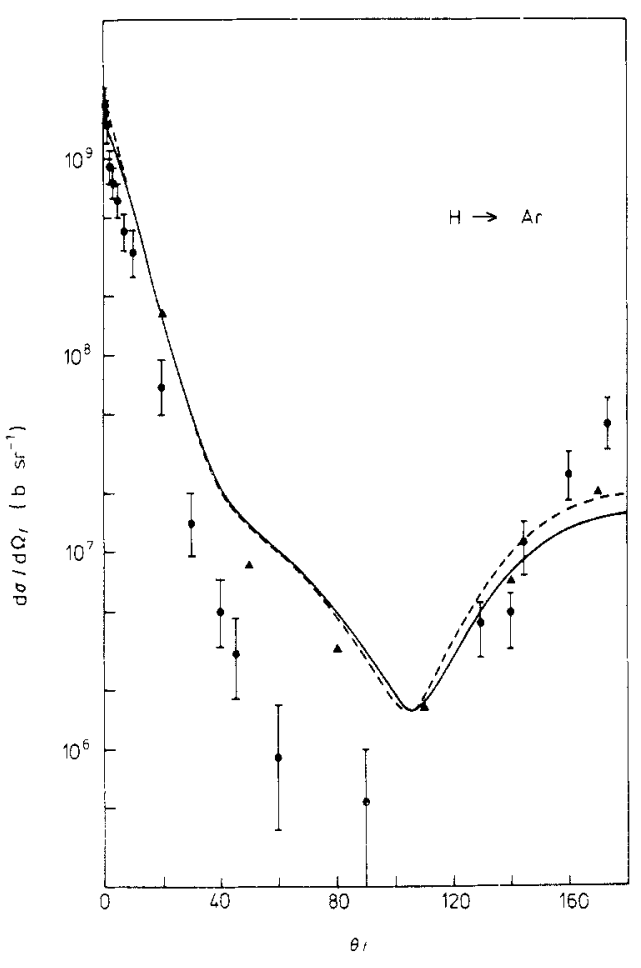

Figure 7. Differential cross section for electron loss in ( $\mathrm{H}, \mathrm{Ar}$ ) collisions at $0.5 \mathrm{MeV}$ as a function of emission angle $\theta_{f}$. The broken curve is a calculation using the elastic electron-target scattering cross section, the full curve includes inelastic effects. The data as well as the results from the elastic scattering model (A) are from Duncan et al (1979).

The experimental energy distribution of the emitted electrons at fixed angle is compared with (and normalised to) the theory in figure 4, and good agreement is found. The peak energy as a function of angle is compared with the theory in figure 5. While this dependence cannot be explained by the elastic scattering model which yields a peak at $\frac{1}{2} m v^{2}$ for all angles, the electron impact approximation is able to reproduce the experimental trend. Unfortunately there exist no data points for intermediate angles to test the theoretical predictions.

One should remark that the electrons ejected around zero degrees $\left(\theta_{f} \leqslant 3^{\circ}\right)$ result from a different process which dominates the charge transfer to the target continuum at $\theta_{f}=0$ (Duncan and Menendez 1977, 1979). Even in highly asymmetric systems as considered here the electron can end up in an eigenstate of the projectile, or an outer target electron may be captured into a continuum projectile state. If the relative velocity $v_{f}$ between electron and projectile is very small, the probability for emission into this final state is proportional to the normalisation constant $2 \pi Z_{1} e^{2} /\left(\hbar v_{f}\right)$ of the Coulomb wave. The transformation to the laboratory frame leads to a divergence given by $\left|\boldsymbol{k}_{f}-m \boldsymbol{v} / \hbar\right|^{-1}$ (Drepper and Briggs 1976), such that this contribution will be important for any system. The energy distribution of these electrons is peaked at $\frac{1}{2} m v^{2}$ and is very narrow. Actually, the shape of the experimental energy distribution at $\theta_{f}=0.4^{\circ}$ with a width much smaller than the one calculated from the electron impact approximation, can be reproduced just by this normalisation factor. The magnitude of this contribution to the electron loss cross section decreases rapidly with angle while the width increases such that it becomes negligible for all angles larger than a few degrees.

To conclude, we have calculated the double-differential cross section for electron loss from light projectiles in asymmetric collisions. By using the electron impact 
approximation we showed that the angular distribution of the emitted electrons is closely related to the angular distribution of electrons elastically scattered from the target with an energy around $\frac{1}{2} m v^{2}$. We demonstrated that it is important to go beyond the Born approximation in the evaluation of the electron-target interaction when calculating the angular distribution as well as the peak position in the energy distribution of the emitted electrons, as long as $\frac{1}{2} m v^{2}$ does not exceed the energy of the electronic excitations in the target. We find qualitative agreement with experiment although the details in the angular distribution cannot be reproduced. Further experimental investigations would help to clarify whether these deviations are accidental or whether they are due to other physical processes.

\section{Acknowledgments}

I should like to thank $\mathrm{K}$ Taulbjerg and $\mathrm{P}$ A Amundsen for their interest in this work.

\section{References}

Bates D R and Griffin G W 1955 Proc. Phys. Soc. A 6890

Betz H D 1972 Rev. Mod. Phys. 44465

Briggs J S 1977 J. Phys. B: Atom. Molec. Phys. 103075

Burch D, Wiemann H and Ingalls W B 1973 Phys. Rev. Lett. 30823

Drepper F and Briggs J S 1976 J. Phys. B: Atom. Molec. Phys. 92063

DuBois R D and Rudd M E 1975 J. Phys. B: Atom. Molec. Phys. 81474

Duncan M M and Menendez M G 1977 Phys. Rev. A 161799 1979 Phys. Rev. A 1949

Fraga S, Karwowski J and Saxena K M S 1976 Handbook of Atomic Data (Amsterdam: Elsevier) p 321 Jakubassa D H and Kleber M 1975 Z. Phys. A 27329

Jhanwar B L, Khare S P and Kumar Ashok Jr 1978 J. Phys. B: Atom. Molec. Phys. 11887

McDowell M R C and Coleman J P 1970 Introduction to the Theory of Ion-Atom Collisions (Amsterdam: North Holland) p 292

Strand T G and Bonham R A 1964 J. Chem. Phys. 401686 